\title{
Erratum
}

\section{The circumstellar structure of the Be shell star $\phi$ Persei}

\section{Modeling}

\author{
W. Hummel ${ }^{1}$ and S. Štefl ${ }^{2}$ \\ 1 European Southern Observatory, Karl-Schwarzschildstr. 2, 85745 Garching, Germany \\ 2 Astronomical Institute, Academy of Sciences of the Czech Republic, 25165 Ondřejov, Czech Republic
}

\author{
A\&A, 368, 471-483 (2001), DOI: 10.1051/0004-6361:20000559
}

\begin{abstract}
We recently modeled the orbital phase variations of the He emission lines of the B2e\&sdO shell binary $\phi$ Per. The angular velocity law used for that study is found to be not correct. We repeated the whole study using a Keplerian velocity law. The application of a Keplerian velocity has only impact on the emission disk radii which are about 50\% larger at $15 R_{k}$ with respect to our previously used velocity law. All other conclusions remain valid.
\end{abstract}

Key words. line: formation - line: profiles - stars: circumstellar matter - stars: emission-line, Be - stars: binaries: spectroscopic

\section{Introduction}

In Hummel \& Štefl (2001, hereafter P2) we modeled phaseresolved emission lines of the Be shell star $\phi$ Per. We could explain the complex emission line variations as due to an external illumination of a circular symmetric disk. We also found emission disk radii of $10 R_{*}$ for all investigated emission lines.

Due to a misinterpretation of the numerical input data for the velocity law, most of the calculated model profiles of P2 are biased and we present here recalculated model profiles and corrected conclusions.

In $\mathrm{P} 2$ we used the values given in Table 2 of Huang (1967) for $\mu=0.1$ labeled "From Three Body Problem" and scaled them to the masses of $\phi$ Per, without taking into account that these values are given in the co-rotating frame, while the proceeding column in Table 2 labeled "From Eq. (19)" are given in the observers frame. Using

$V_{\mathrm{K}}^{\mathrm{co}-\mathrm{rot}}+r=\sqrt{\frac{1-\mu}{r}}=V_{\mathrm{K}}^{\mathrm{obs}}$

the values of "From Three Body Problem" column can be easily expressed in the observers frame to be compared with the "From Eq. (19)" column.

As a consequence of our misinterpretation the fake linear correction factor taken from the numerical tables (see Eq. (1) of P2) simply reflects the transformation between co-rotating frame (co-rotating with orbital period of the binary) and the

Send offprint requests to: W. Hummel, e-mail: whummel@eso.org
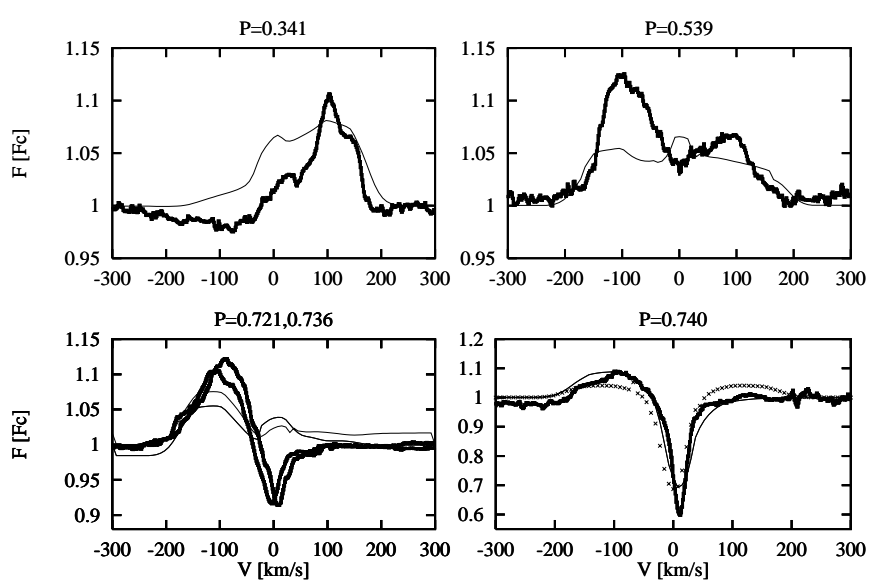

Fig. 1. Fit of the He I 5876 emission feature at four different orbital phases. Model parameters are given in Table 1. The dotted line in the figure for $P=0^{\mathrm{p}} .74$ is the model profile for phase $0^{\mathrm{p}} .0$.

observers frame. The radial run of the rotation in a disk embedded in a binary is in fact much closer to Keplerian. The applied angular velocity is underestimated by $24 \%$ at $10 R_{*}$ with respect to the Keplerian value and hence the emission disk radii are underestimated as well.

We re-investigated the emission line variations using the same model construction, but used a Keplerian rotation velocity law with the parameter $j=0.5$ (line 1 in Fig. 1 of P2) instead, since the difference between the Keplerian velocity law and the values based on the three body calculations are rather 

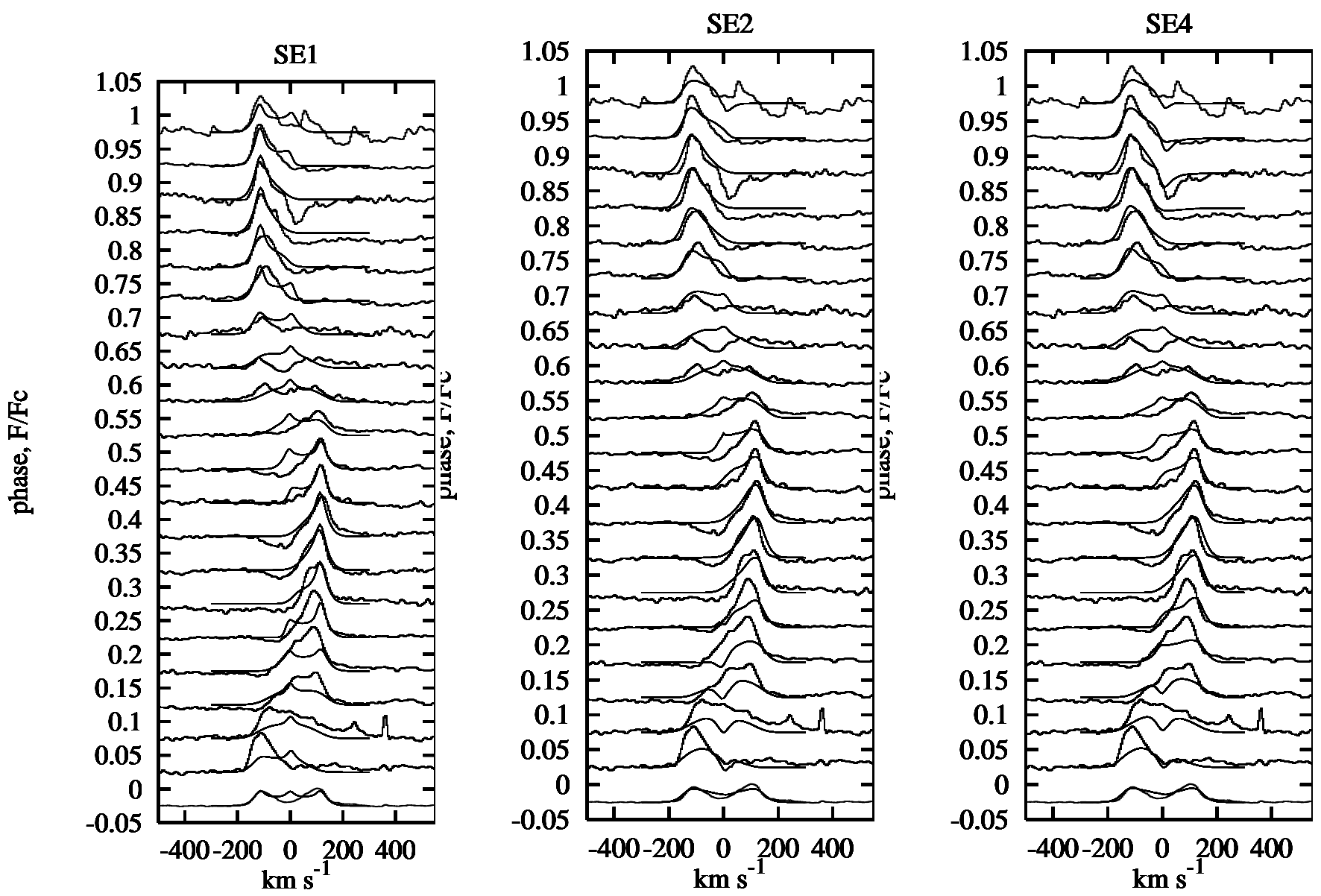

Fig. 2. Bold profiles: phase resolved observations of He I 6678 emission lines, corrected for phase shifted stellar absorption and phase binned. The lowermost line profile is the orbital mean profile. Details on the observations are given in P1. Thin: phase dependent theoretical emission line profiles for He I 6678. SE1) The sector model. SE2): as SE1), except the shell absorption is taken into account in the model profiles. SE4): as SE2), except the differential rotation is taken into account for the outgoing boundary $\phi_{2}$ as well as for the incoming boundary $\phi_{1}$ to test the preceding shell absorption. Parameters for all three models SE1, SE2 and SE4 are given in Table 1.

small. The following models have been recalculated: SE1, SE2, SE3, and SP3 for of He I 6678 and SP5 for He I 5876.

\section{Consequences}

\subsection{Fe $/ / 5317$}

Model C2 of P2 is not applicable and model $\mathrm{C} 1$ for which we used Keplerian rotation with an emission disk radius of $R_{\mathrm{d}}=12$ is right.

\section{2. $\mathrm{He} / 6678$}

Selected new best fit models are given in Fig. 2 and corresponding best fit values are given in Table 1 . The corrected velocity law results in a new emission disk radius for He I 6678 of $R_{\mathrm{d}}=15$. The opening angle in the sector model is retained and the excitation radius in the sphere model is slightly enlarged.

The velocity law has no impact on the diagnostic method using the peak-velocity diagram.

Our arguments concerning the advection are also not affected, since the gas movement at the outer rim of the disk has been neglected for the short revolution time of $\simeq 20 \mathrm{~h}$ at $R_{*}$.
The angular distribution of the emission region in the disk is retained for Keplerian rotation, except that it is now extended up to $R_{\mathrm{d}}=15$.

\section{He I 5876}

The shape of the He I 5876 emission region becomes more concentrated to the outer rim of the disk and the model profiles do less agree with the observations, especially for shell absorption at $P=0.7$.

\section{Discussion}

The repeated model calculations have shown that except the emission disk radius all conclusions of $\mathrm{P} 2$ are valid. The Fe II 5317 emission disk radius of $R_{\mathrm{d}}=12$ and the newly derived radius of $R_{\mathrm{d}}=15$ for He I 6678 and 5876 is larger with respect to our previous biased calculations. It is now of the order of the Roche radius $R_{\mathrm{R}}=16.8$. The derived disk emission radii agree better with those derived by Hummel \& Vrancken (1995).

Acknowledgements. We thank Atsuo Okazaki for pointing out the errors concerning the rotation law. 
Table 1. Final best-fit parameters for the net emission line profile.

\begin{tabular}{lllllllll}
\hline \hline \multicolumn{7}{c}{ He I 6678- sector model } \\
\hline model & $n_{0}$ & $m$ & $S^{\mathrm{L}}$ & $R_{\mathrm{i}}$ & $R_{\mathrm{d}}$ & $\Delta \phi$ & $\phi_{2}-\phi_{1}$ & $\chi^{2}$ \\
\hline SE1 & $1.8 \mathrm{e}-5$ & 0.5 & 1.00 & 5.1 & 14.9 & .135 & 111 & $2.10 \mathrm{e}-3$ \\
$\mathrm{SE} 2$ & $7.5 \mathrm{e}-2$ & 0.5 & $5 \mathrm{e}-4$ & 5.0 & 15.3 & .135 & 97 & $2.33 \mathrm{e}-3$ \\
$\mathrm{SE}^{a}$ & $8.0 \mathrm{e}-2$ & 0.5 & $5 \mathrm{e}-4$ & 5.0 & 15.3 & .135 & 112 & - \\
\hline \hline
\end{tabular}

He I 6678 - sphere model

\begin{tabular}{lllllllll}
\hline model & $n_{0}$ & $m$ & $S^{\mathrm{L}}$ & $R_{\mathrm{i}}$ & $R_{\mathrm{d}}$ & $\Delta \phi$ & $R_{\mathrm{e}}$ & $\chi^{2}$ \\
\hline SP2 & $4.0 \mathrm{e}-2$ & 0.5 & $5 \mathrm{e}-4$ & 1.0 & 14.7 & 0.0 & 27.8 & $2.3 \mathrm{e}-3$ \\
\hline \hline \multicolumn{7}{c}{ He I 5876 - sector model } \\
\hline model & $n_{0}$ & $m$ & $S^{\mathrm{L}}$ & $R_{\mathrm{i}}$ & $R_{\mathrm{d}}$ & $\Delta \phi$ & $\phi_{2}-\phi_{1}$ & $\chi^{2}$ \\
\hline $\mathrm{SE}^{b}$ & 0.63 & 0.5 & $1 \mathrm{e}-3$ & 12 & 14.6 & 0.0 & 210 & $4 \mathrm{e}-1$ \\
\hline
\end{tabular}

Notes:

${ }^{a}$ A phase shift of $2 \pi$ is used for both boundaries $\phi_{1}$ and $\phi_{2}$; the shadow radius $R_{\mathrm{o}}=8.6 R_{*}$.

${ }^{b}$ A phase shift of $0.8 * 2 \pi$ is used for both boundaries $\phi_{1}$ and $\phi_{2}$.

\section{References}

Huang, S. S. 1967, ApJ, 148, 793
Hummel, W., \& Vrancken, M. 1995, A\&A, 302, 571 (HV1)

Hummel, W., \& Štefl, S. 2001, A\&A, 368, 471 (P2)

Stefl, S., Hummel, W. \& Rivinius, Th. 2000, A\&A, 358, 208 (P1) 